\title{
Use of ANSYS Software for the Acetabular Cup Structure Analysis out of the Hip Implant
}

\author{
A. RASHWAN \\ PhD Student \\ Department of Mechanical Engineering, Faculty of Engineering, University of Debrecen \\ Doctoral School of Informatics, Faculty of Engineering, University of Debrecen
}

\begin{abstract}
Modelling the hip implant has been one of the most important researches over the past few decades. In addition, using the ANSYS software for this purpose is well-known procedure to understand the real reaction of the hip implant parts during the daily life of the installed part. This study is to focus on the practical part of the use of ANSYS software to analyse the performance of the hip implant through the feature of structure analysis available in the ANSYS. The research applies the static loads behaviour only with the help of the static structural analysis to view the advantages and the disadvantages of every design, which helps us estimate the implant's behaviour. The study investigates the optimization of the acetabular cup using the lattice optimization along with the infill option available in the ANSYS software in order to optimize the stress and the fixture of the cup inside the pelvis.
\end{abstract}

\section{Introduction}

Some of the bone diseases force the patient to have the hip replaced with an artificial implant [1]. Researchers are trying to extend the age of the THR total hip replacement with the least number of defects that may occur during the patient's life. The normally accepted lifespan of the implant is about 15 years [2]. Most of the studies focus on avoiding the defects happening, which gives the implant the long life required.

There are certain diseases that affect the performance of any implants installed such as loosening, dislocations and the contact pressure between the acetabular cup and the implant itself [3]. Researchers have invented the term 'impingement' to describe the unnecessary contact between the acetabular cup and the head of the femur. They believe that the recurrent impingement might cause the failure of the joint [4].

Many studies have mentioned some of the parameters responsible for having a durable acetabular component such as the size of the femoral head the head-neck diameter ratio and the component orientation [5]. ROM range of motion also has become one of the important indicators that can tell us how successful the joint is, every manufacturing company, however has set their own ROM for the joints they make [6].

Most of the above-mentioned diseases are related to the static loads that happen over time with the constant wear and tear of the joint. The IDAC Integrated Design and Analysis Consultants use ANSYS software in order to understand the necessary implantation and the installation forces required for the 
variety of the replacements available for every patients. The ANSYS can create parametric models that help evaluate the cup geometry. The finite element studies performed on the cup can give us a good estimate on the relationship between the proposed forces/constraints and the stress generated accordingly. The idea of previously testing the joints and the implants through the ANSYS software can help save a great deal in comparison to the physical testing [7] since ANSYS has been used before for tests like compression and stress [8].

One of the important factors to watch after the acetabular revision surgery is the effect of the load on the cup, because the stability of the cup and its integration with the body is something necessary to achieve. Due to the complexity of the shapes, whether it is the pelvis parts or the acetabular cup, FE the finite element modelling plays a major role in evaluating the stress distribution in the hip prosthesis [9].

The topology optimization TO has helped the designers to minimize the implant failure probability, thus increased the reliability of the prosthesis. The main aim of the manufacturers with the FEM and the topology optimization is to reduce stress, which is taking place on the prosthesis parts in general and on the acetabular cup in particular. In addition, the topology optimization feature is utilized for the static finite element analysis of the implant, which helps reduce the stress shielding. Using the ANSYS software topology optimization will redesign the whole implant shape, give a suggestion based on the software algorithm, and provide reconstruction idea for the implant geometry that helps optimize it and, at the same time, keep the durability required [10].

Along with the study of the structural analysis, many researches have looked into optimising the implant in general and the acetabular cup in particular. ANSYS was used to optimise hip implant in order to minimise the stress shield cost and time of the prosthesis manufacturing. Mathheck et al. 1990 tried to optimize the hip implant with the FEM process and discovered that a hollow geometry of the stem is able to decrease the stress in a reasonable amount [11].

Based on the medical recommendations, the cubic unit cell can be applied in order to prepare for the FEM analysis to point the applicable load on an implant [12-16], which is why this research will give the cubic unit cell lattice a try in order to explore the effect of such a type of structure.

ANSYS software has been used before for the FEM analysis and test of specimen

\section{Methods}

\subsection{Static Structural ANSYS}

For the case in this study, we have chosen a medium-size cup for the acetabular cavity as shown in the following figure 1, the file was imported in STP format after being drawn using a CAD program. The meshing size is set to default with 0 -element size. 

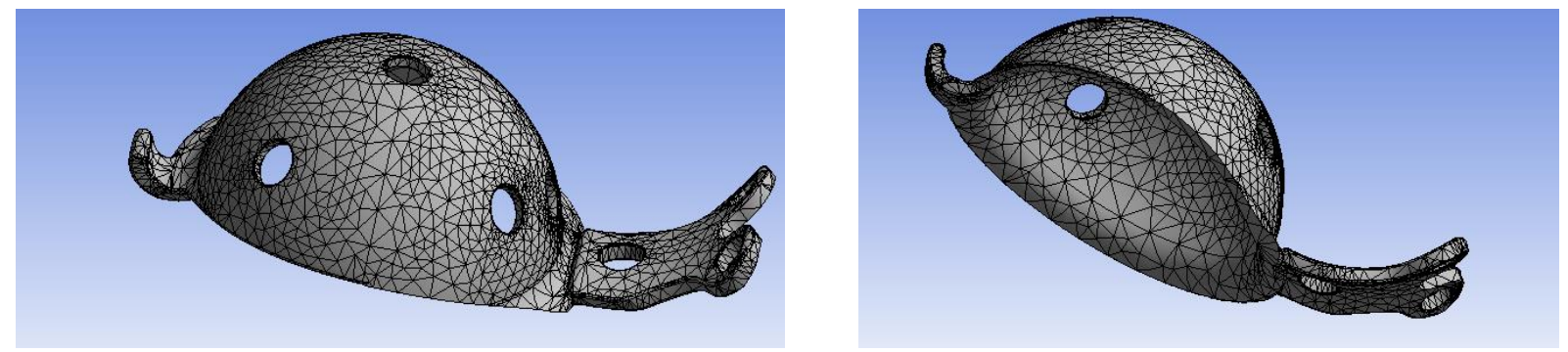

Figure 1. the acetabular cup

The fixture 'fixed support' is applied on the outer face of the dome in the cup considering the face will be glued to the pelvis. The shape shown in the figure 1 has extra part to stick well to the pelvis. The inner side of the cup receives the pressure from the femoral head during the real life activities that exert loads to be considered.

The applied pressure in the experiment is around $750 \mathrm{lb}$. (350 kg approximately) and the radius of the cup is around $3.5 \mathrm{~cm}$ so the pressure generated on the surface will be around $490 \mathrm{~Pa}$. The ANSYS offers the equivalent stress (von-mises) and the total deformation charts. The next two figures 2, 3 preview both stress charts:

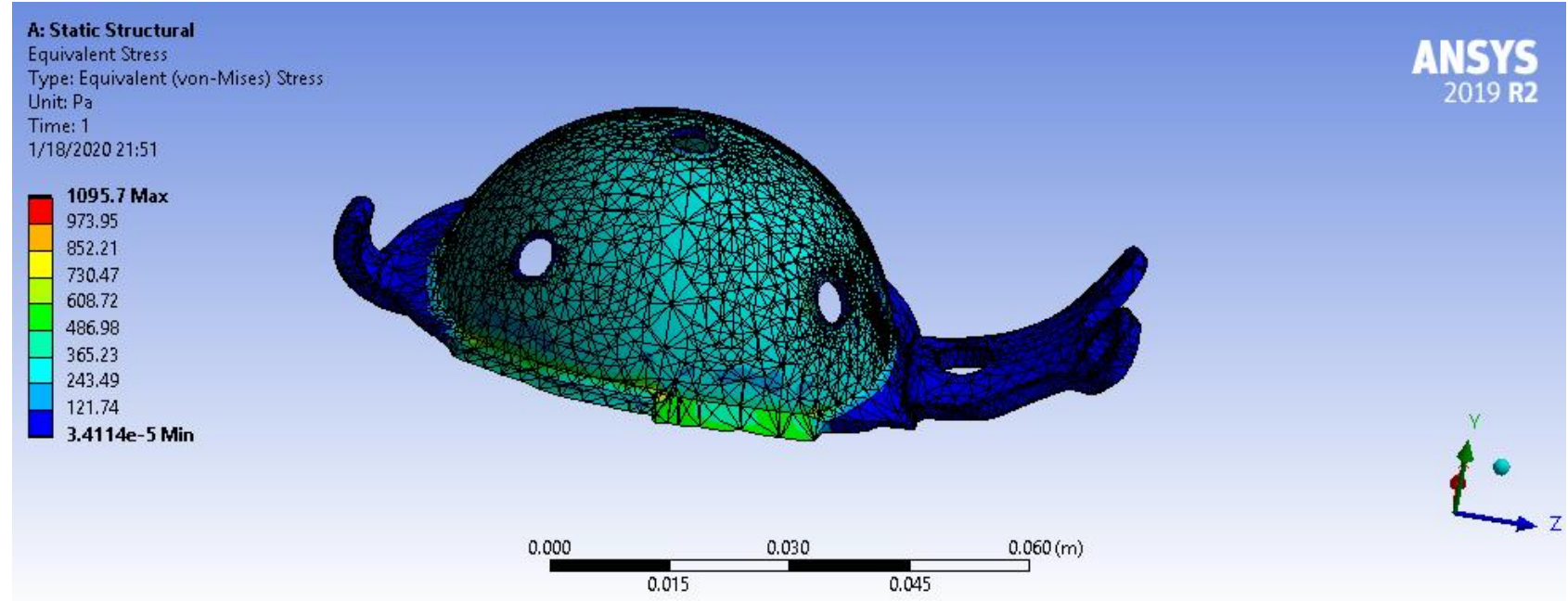

Figure 2. Von-mises stress chart, ANSYS

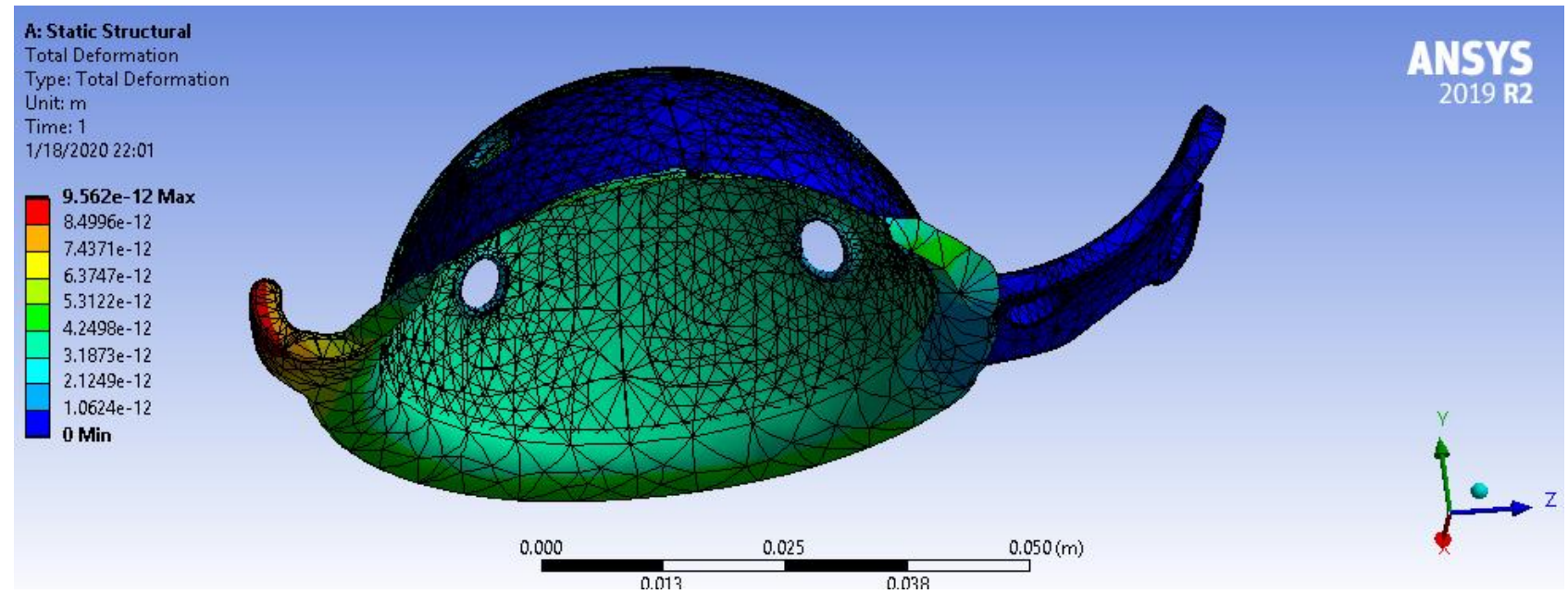

Figure 3. total deformation 


\subsection{Topology optimization}

Many studies followed in order to reduce the stress shielding and to optimize the hip implant. In our study, we are trying to focus on the acetabular cup optimization using the same case that we investigated in the previous section as a structural analysis.

After having the cup analysed through the static structural feature, the ANSYS can apply the topology optimization feature in order to optimize it, whether the optimization is about reducing mass, volume, or even stress. One of the new options in the ANSYS 2019 is the lattice optimization and the infill feature in the SpaceClaim. The infill feature can decorate the surface of the cup in order to get a rough surface that can stick to the pelvis and allow the body to build a new structure of bone cells into the cup, which makes the cup more fixed over time.

The following figure 4 shows the topology optimization tree in ANSYS. It demonstrates how to start a topology optimization study out of the previous results.
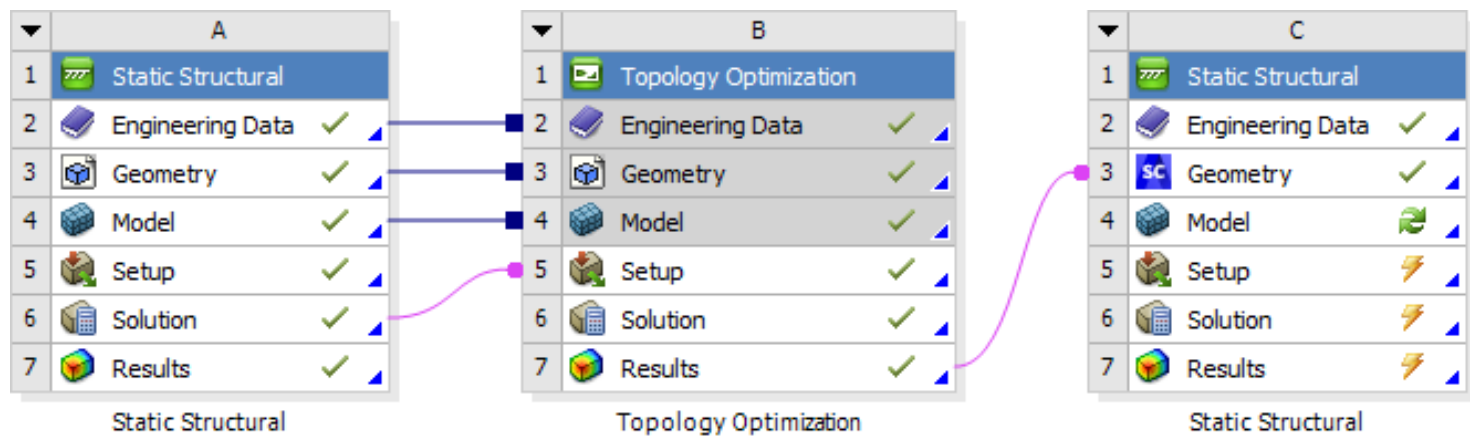

Figure 4. topology optimization

However, our focus on this part of the topology is the lattice optimization using the infill option in the SpaceClaim as mentioned before. To use the topology optimization results as a new geometry as shown in figure 4, we can open a new static structural window and use the results there, and then we apply the infill feature choosing a certain type of structure. The software ANSYS offers many types of unit cells as shown in the next figure 5 :

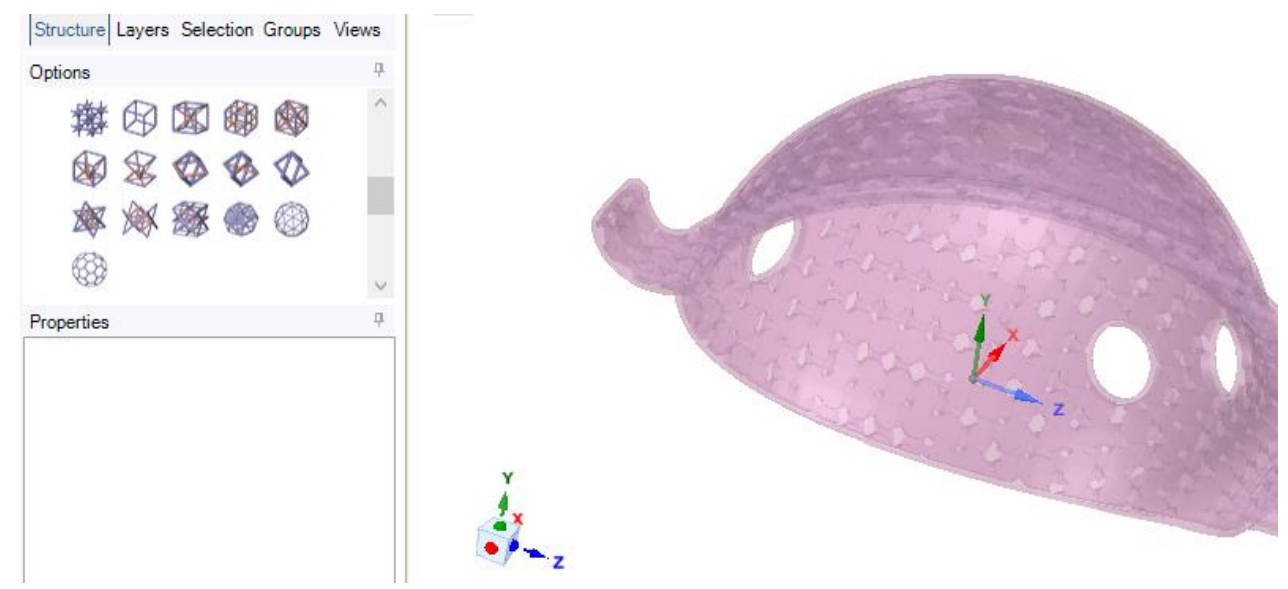

Figure 5. unit cells options in lattice feature in SpaceClaim 
The study uses the cube-lattice option with removing the outer surface of the cup, which exposes the inner structure of the cup into the pelvis once connected. The following figure 6 shows the outcome file acquired from applying such a feature.

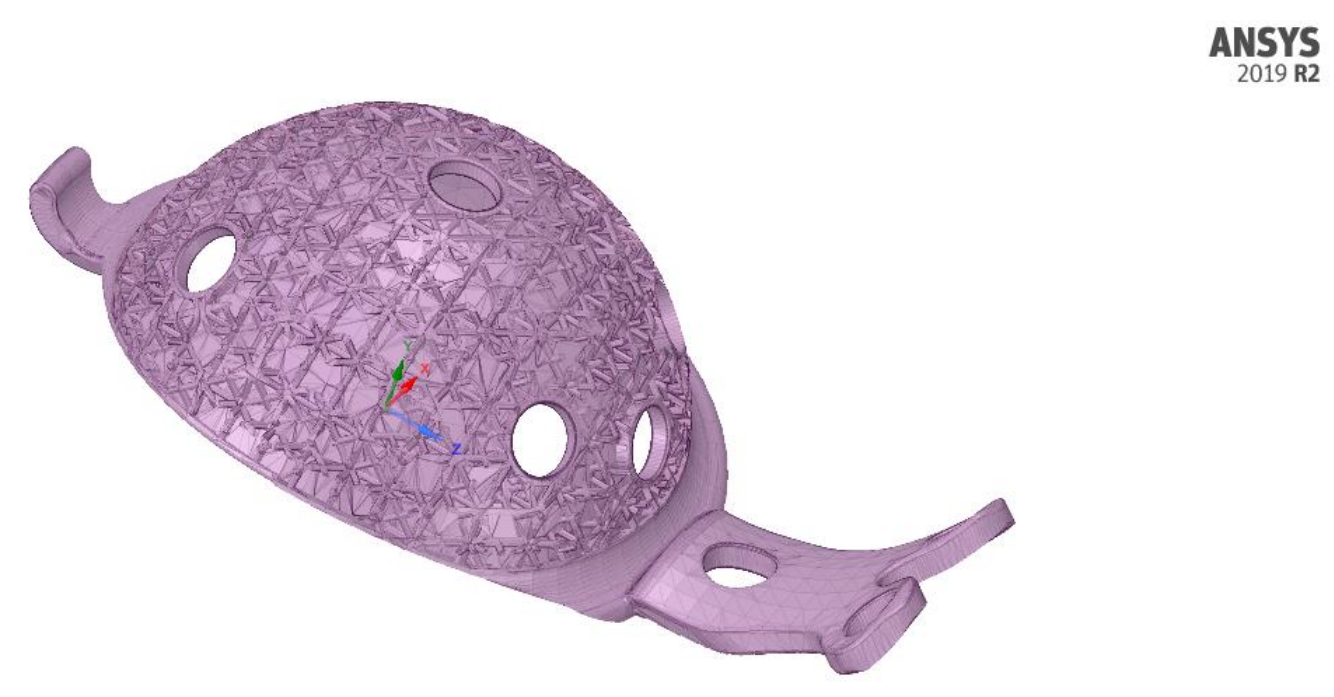

Figure 6. the lattice-shell option applied to the structure of the acetabular cup

\section{Conclusion:}

Using the ANSYS software shows two advantages during the study. The first option is the static structural option and getting to analyse the prosthesis with the FEM analysis available in order to anticipate the weaknesses and the strengths in the prosthesis design before moving on to the manufacturing process.

The second option to get benefit from in the software is the topology optimization option, which allows the designer to optimize the initial design proposed, and enables the user to repair the problems that arise through time with the patient using the implant. With the previous section, we have noticed how the optimized part with the lattice option helped fix the cup into the pelvis with the rough surface that is ready to have bone built on it through time.

\section{References}

[1] L. Mattei, F. Di Puccio, B. Piccigallo, and E. Ciulli, "Lubrication and wear modelling of artificial hip joints: A review," Tribology International 44 (5), 532-549 (2011).

[2] S. Shankar, L. Prakash, and M. Kalayarasan, "Finite Element Analysis of Different Contact Bearing Couples for Human Hip Prosthesis," International Journal of Biomedical Engineering and Technology 11 (1), 66-80, (2013).

[3] M. Ghaffari, R. Nickmanesh, N. Tamannaee, and F. Farahmand, "The impingement-dislocation risk of total hip replacement: effects of cup orientation and patient manoeuvres.," Conference proceedings : IEEE Engineering in Medicine and Biology Society 202 (1), 6801-6804 (2012). 
International Journal of Engineering and Management Sciences (IJEMS) Vol. 5. (2020). No. 2

DOI: 10.21791/IJEMS.2020.2.1.

[4] K.-H. Widmer and B. Zurfluh, "Compliant positioning of total hip components for optimal range of motion.," Journal of orthopaedic research : official publication of the Orthopaedic Research Society 22 (4), 815-821 (2004).

[5] S. Shuib, B. B. Sahari, W. S. Voon, M. Arumugam, and A. H. Kadarman, "Short Communication Stress Analysis of Femoral Hip with Bone Resorption," Trends Biomaterial Artifical Organs 27 (2), 88-92 (2013).

[6] F. Yoshimine, "The safe-zones for combined cup and neck anteversions that fulfil the essential range of motion and their optimum combination in total hip replacements.," Journal of Biomechanics 39 (7), 1315-1323 (2006).

[7] J. Thakker, "Hip to Simulation Evaluation of designs for a hip replacement prosthesis overcomes physical and scientific limitations. Integrated Design and Analysis Consultants, U.K.

[8] D. Huri, T. Mankovits, "Comparison of the material models in rubber finite element analysis." IOP Conference Series: Materials Science and Engineering, 393 Paper: 012018 (2018).

[9] J. Stolk, S.A. Maher, N. Verdonschot, P.J. Prendergast, R. Huiskes, "Can finite element models detect clinically inferior cemented hip implants?" Clin Orthop Relat Res.;409:138-150. (2003).

[10] M. Fraldi, L. Esposito, G. Perrella, A. Cutolo, S.C. Cowin, "Topological optimization in hip prosthesis design" Biomechanics and Modeling in Mechanobiology, volume 9, pages 389-402 (2010).

[11] M.I.Z. Ridzwan, S. Shuib, A.Y. Hassan, A.A. Shokri, M.N.M. Ibrahim, "Optimal design of femoral hip prosthesis using topology optimization to reduce stress shielding" Journal of Applied Sciences 6(13), pp. 2768-2773 (2006).

[12] T. Mankovits, "Numerical analysis of unit cell models for orthopedic applications" IOP Conference Series: Materials Science and Engineering 393 Paper: 012019 (2018).

[13] D. Huri, T. Mankovits, "Automotive rubber part design using machine learning" IOP Conference Series: Materials Science and Engineering 659 pp. 1-6. Paper: 012022 , 6 p. (2019).

[14] T. Mankovits, T.A. Varga, S. Manó, I. Kocsis, "Compressive Response Determination of Closed-Cell Aluminium Foam and Linear-Elastic Finite Element Simulation of $\mu C T$-Based Directly Reconstructed Geometrical Models" Strojniski Vestnik-Journal of Mechanical Engineering 64(2), pp. 105-113., 9 p. (2018).

[15] T. Mankovits, I. Budai, G. Balogh, A. Gábora, I. Kozma, T.A. Varga, S. Manó, I. Kocsis, "Structural analysis and its statistical evaluation of a closed-cell metal foam" International Review of Applied Sciences and Engineering $5: 2$ pp. 135-143. , 9 p. (2014).

[16] T. Mankovits, T. Szabó, I. Kocsis, I. Páczelt, "Optimization of the Shape of Axi-Symmetric Rubber Bumpers" Strojniski Vestnik-Journal of Mechanical Engineering 60(1), pp. 61-71., 11 p. (2014). 